\title{
Alkaline-stable nickel manganese oxides with ideal band gap for solar fuels photoanodes
}

Santosh K. Suram, Lan Zhou, Aniketa Shinde, Qimin Yan, Jie Yu, Mitustaro Umehara, Helge S. Stein, Jeffrey B. Neaton, and John M. Gregoire

\section{X-ray diffraction}

The crystal structures and phase distribution of the composition libraries were determined through Xray diffraction (XRD) measurements with the x-ray spot size limited to a $1 \mathrm{~mm}$ length scale, over which the composition is constant to within approximately $1 \%$. For the $\mathrm{Ni}_{x} \mathrm{Mn}_{1-\mathrm{x}} \mathrm{O}_{2}$ libraries, a series of measurements across the composition gradient was used to generate a structural phase map to elucidate structure-property relationships. XRD patterns were acquired with a Bruker DISCOVER D8 diffractometer with $\mathrm{Cu} \mathrm{K}_{\alpha}$ radiation from a Bruker $\mathrm{I} \mu \mathrm{S}$ source (Bruker AXS Inc., Madison, WI). Using a 0.5 $\mathrm{mm}$ collimator, the effective thin film measurement area was approximately $0.5 \times 1 \mathrm{~mm}$. Diffraction images were collected using a two-dimensional VÅNTEC-500 detector and integrated into onedimensional patterns using DIFFRAC.SUITE ${ }^{\mathrm{TM}}$ EVA software. For patterns in which multiple crystalline phases were identified, the relative phase fraction of each phase was approximated by normalizing the measured intensity of the most distinguishing peak of each phase.

\section{Composition measurements}

X-ray fluorescence (XRF) was employed to measure the composition of the thin films library. The measurements were performed on an EDAX Orbis Micro-XRF system (EDAX Inc., Mahwah, NJ) with an Xray beam approximately $2 \mathrm{~mm}$ in diameter. XRF counts at a certain x-ray energy directly gives an indication of the amount of material, but to be able to get the composition in atomic percent (at.\%), the composition quantification was calibrated using the energy-dispersive $x$-ray spectroscopy (EDS). The EDS measurements were performed using an Oxford Instruments X-Max (Oxford Instruments, Concord, MA) detector on a FEI Nova NanoSEM 450 (FEI, Hillsboro, OR) with excitation from a $12 \mathrm{keV}$ electron beam and the composition quantification was obtained using a thin film model in the INCAEnergy EDS analysis software.

The XRF characterization of the libraries not only established the composition axes in Figures 1 and 2 but also provided an estimate of the metal oxide thickness. In Figure 1, the circled composition with $\mathrm{Ni} /(\mathrm{Ni}+\mathrm{Mn})$ values of 0.48 and 0.69 are approximately 195 and $220 \mathrm{~nm}$ thick, respectively. In Figure 2, the 0.48 and 0.53 samples are approximately 131 and $129 \mathrm{~nm}$ thick, respectively.

\section{Experiment Details}

A photograph of the sputter deposited library, electrochemical stability data, and optical characterization of $\mathrm{Ni}_{6} \mathrm{MnO}_{8}$ are provided in Figures S1-S3. The LEDs used for PEC measurements are summarized in Table S1. All PEC measurements are performed in an under filled-type cell where the electrolyte contact area is larger than the illumination area, which for the measurements with FCN corresponds to the photocurrent values being a lower limit as the not-illuminated portion of the working electrode acts as a partial current shunt. These are all high irradiance measurements intended to 
elucidate trends in photoactivity as a function of composition and phase, or as in the case of Figure S2 to rapidly assess stability. This 30 minute stability measurement included approximately 900 illumination cycles, and the total photocurrent passed was in excess of $0.3 \mathrm{mC} \mathrm{cm}^{-2}$, which corresponds to in excess of $300 \mathrm{nmol} \mathrm{cm}^{-2}$ of fundamental charge. If either $\mathrm{Ni}$ or $\mathrm{Mn}$ was corroding by a $1 \mathrm{e}^{-}$process, this charge would have resulted in loss of half of the respective element from the thin film, for which there is no evidence. The lack of corrosion in PEC experiments is more directly evident by the lack of photocurrent in the measurements without a sacrificial hole acceptor, since photocorrosion processes are generally not altered by such electrolyte additives.

Both $\mathrm{NiMnO}_{3}$ and $\mathrm{Ni}_{6} \mathrm{MnO}_{8}$ phases were identified in the recently-reported computational search of screening Manganese-based ternary oxide photoanodes. ${ }^{1}$ Table 1 of that work shows that $\mathrm{NiMnO}_{3}$ was determined to be non-photoactive at the OER Nernstian potential by the PEC experiments in $\mathrm{pH} 6.6, \mathrm{pH}$ 10 and $\mathrm{pH} 13$ electrolytes, commensurate with the present work where the redox couple or hole acceptor are necessary to obtain photoactivity (Figure 1c showing photocurrent with FCN redox couple but not in its absence, Figure 2 showing photocurrent with sulfite hole acceptor).

To more clearly elucidate the relationship between transient and quasi-stead state photocurrent for the 2 samples in Figure 2b, a zoomed in version of the last 3 illumination cycles are shown in Figure S4, where the transient and quasi-steady-state photocurrent are illustrated to show that the addition of $\mathrm{Ni}_{6} \mathrm{MnO}_{8}$ to $\mathrm{NiMnO}_{3}$ alleviates an apparent issue with surface state population, converting more of the absorbed photons to photocurrent in steady state.

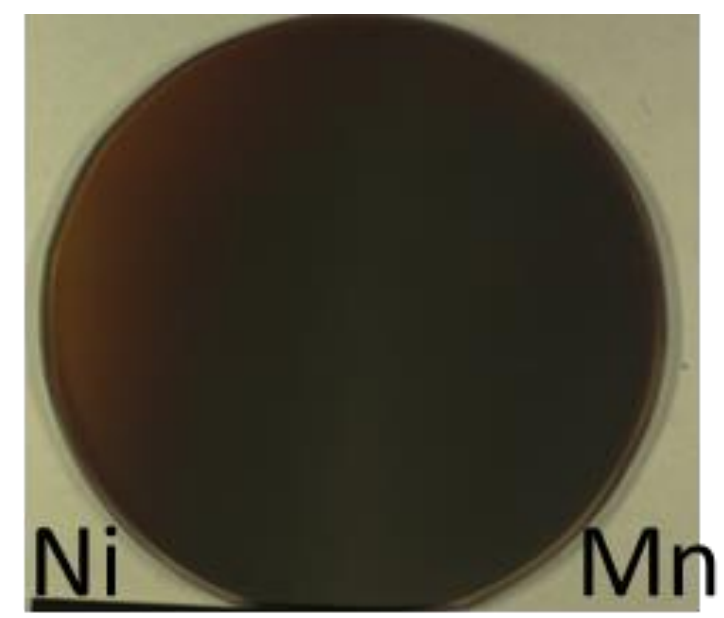

Figure S1. An image of the Ni-Mn-O library on glass substrate after annealing, demonstrating the high optical density of the thin film compositions. The elemental symbols indicate the locations of the sputtering sources. 

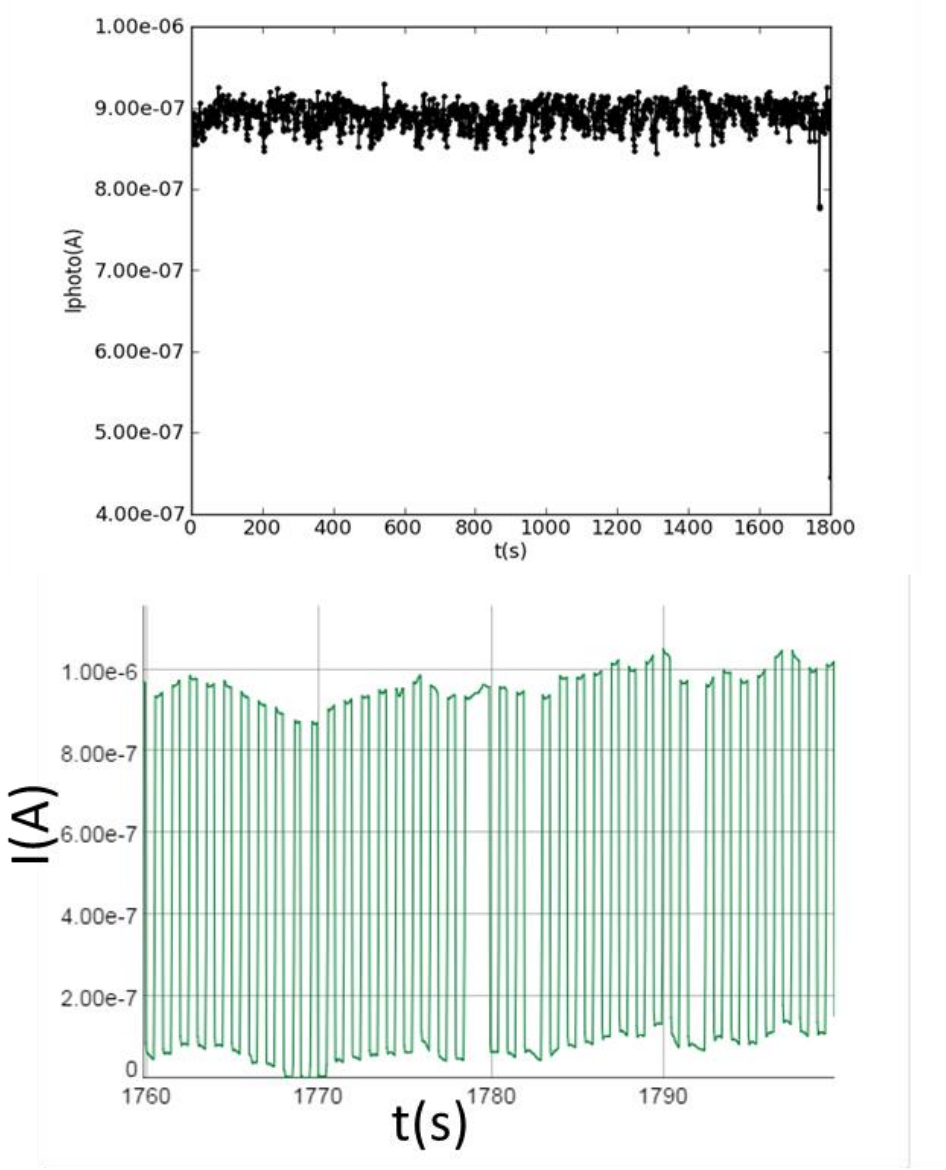

Figure S2. (top) Photocurrent with ferri/ferro cyanide (FCN) redox couple for $\mathrm{Ni}_{0.48} \mathrm{Mn}_{0.52}$ composition as a function of time measured over 30 minutes. (bottom) Current as a function of time for the same sample over the last 40 seconds of the 30 minute measurement, where the couple illumination cycles that appear to be a different duration are from a timing delay artifact of the instrument control software and are not impactful for the characterization of photocurrent stability. 

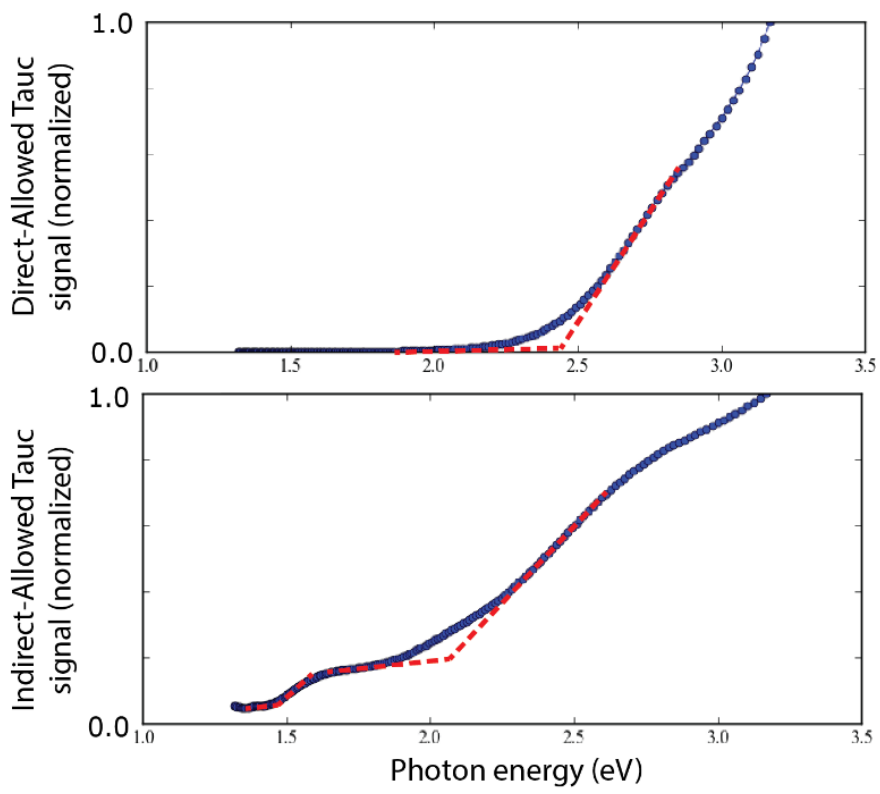

Figure S3. Tauc signals for Direct (top) and Indirect (bottom) allowed transitions for a sputter deposited $80 \%$ phase pure $\mathrm{Ni}$ $\mathrm{MnO}_{8}$ thin film. Linear models of the baseline and above-gap absorption are shown in dashed red lines, indicating a clear direct band gap at $2.4 \mathrm{eV}$ and that a lower indirect gap likely exists, which could be approximately 1.5 or $2.1 \mathrm{eV}$.

Table S1: Summary of LED sources used for PEC experiments. The "front" and "back" refer to illumination from the same side as the electrolyte contact and same side as the electrical back contact, respectively.

\begin{tabular}{|c|c|c|c|c|c|}
\hline $\begin{array}{c}\text { LED } \\
\text { Model/Geometry }\end{array}$ & $\begin{array}{c}\text { Wavelength } \\
\text { (nm) }\end{array}$ & $\begin{array}{c}\text { Energy } \\
(\mathrm{eV})\end{array}$ & $\begin{array}{l}\text { Illum. Area } \\
\left(10^{-3} \mathbf{c m}^{2}\right)\end{array}$ & $\begin{array}{l}\text { Irradiance } \\
\left(\mathrm{mW} / \mathrm{cm}^{2}\right)\end{array}$ & Figures \\
\hline ThorLabs M385F1/front & $385 \pm 5$ & $3.22 \pm 0.04$ & 6.4 & 439.3 & $1 \mathrm{c}\left(J_{O E R}\right)$ \\
\hline ThorLabs M385F1/front & $385 \pm 5$ & $3.22 \pm 0.04$ & 18 & 204.2 & $2 b$ \\
\hline ThorLabs M385F1/back & $385 \pm 5$ & $3.22 \pm 0.04$ & 25 & 181.2 & $1 \mathrm{~b}, 1 \mathrm{c}\left(J_{F C N}\right), 3 \mathrm{a}$ \\
\hline ThorLabs M455F1/back & $455 \pm 12.5$ & $2.72 \pm 0.08$ & 25 & 124.0 & $3 a$ \\
\hline ThorLabs M530F1/back & $530 \pm 16.5$ & $2.34 \pm 0.07$ & 25 & 56.8 & $3 a$ \\
\hline ThorLabs M617F1/back & $617 \pm 9$ & $2.01 \pm 0.03$ & 25 & 117.6 & $3 a$ \\
\hline
\end{tabular}




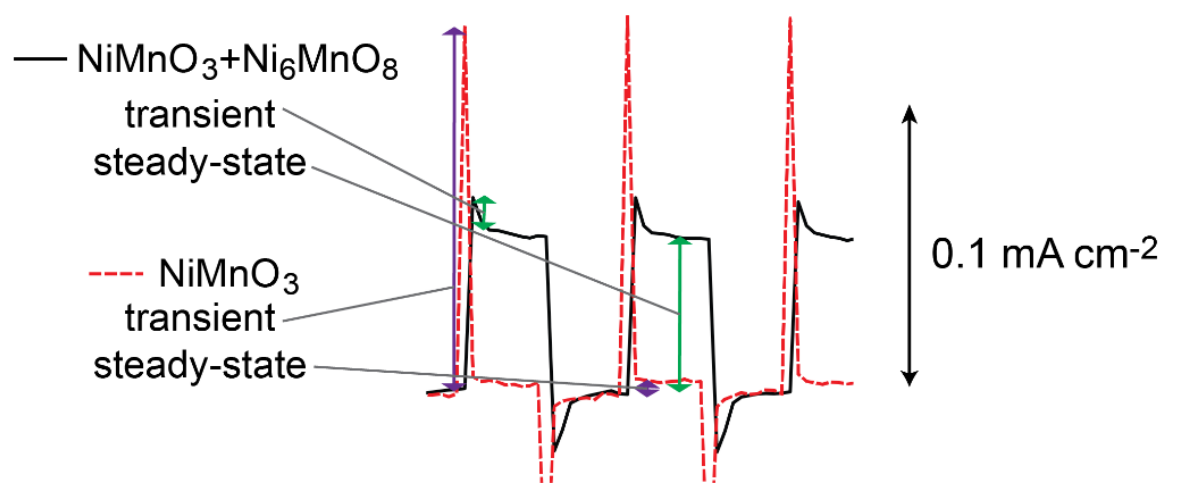

Figure S4. Zoomed-in version of Figure $2 \mathrm{~b}$ to enable labelling of the transient and quasi-steady-state photocurrent for of $\mathrm{NiMnO}_{3}$ (purple arrows) and $\mathrm{NiMnO}_{3}+\mathrm{Ni}_{6} \mathrm{MnO}_{8}$ (green arrows).

\section{SEM imaging}

Secondary Electron Microscopy (SEM) were performed with a FEI Nova NanoSEM 450 using a $15 \mathrm{keV}$ electron beam for the 2 samples measured in sulfite-containing electrolyte in Figure 2 . The images in Figure S5 (note the slightly different magnification and scale bars) show that the morphology is similar in both samples with apparent columnar-like morphology on the $200 \mathrm{~nm}$ scale. The invariance of the morphology upon addition of the $\mathrm{Ni}_{6} \mathrm{MnO}_{8}$ phase to the $\mathrm{NiMnO}_{3}$ further corroborates the conclusion that a heterojunction gives rise to the increased steady state photocurrent.
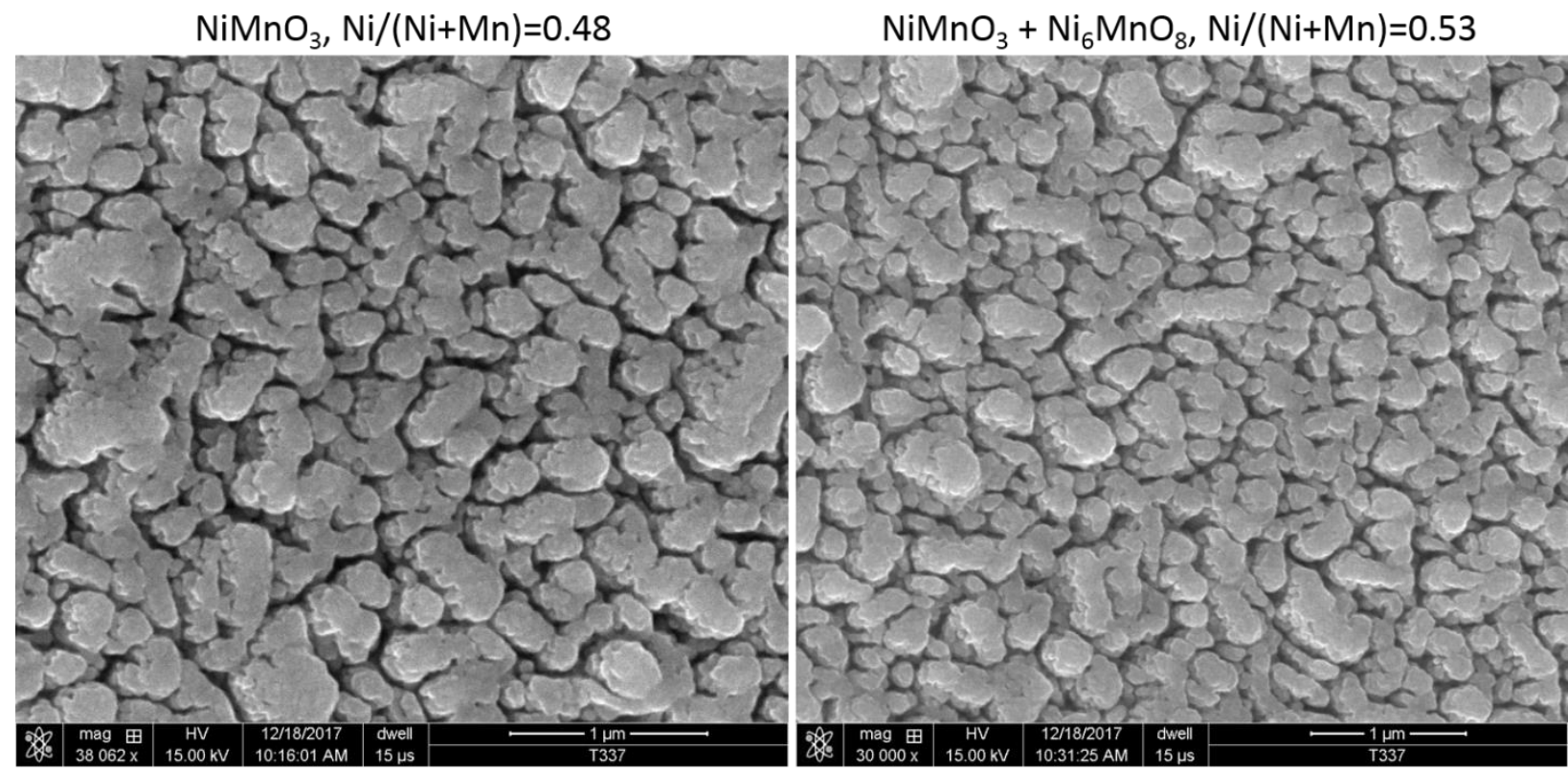

Figure S5. SEM images representative of the 2 samples in Figure 2: $\mathrm{Ni} /(\mathrm{Ni}+\mathrm{Mn})$ values of $0.48\left(\mathrm{NiMnO}_{3}\right)$ and $0.53\left(\mathrm{NiMnO}_{3}+\right.$ $\mathrm{Ni}_{6} \mathrm{MnO}_{8}$ ) with approximately thickness 131 and $129 \mathrm{~nm}$, respectively.

\section{Additional electrochemical characterization}

To further elucidate the properties of the Ni-Mn oxide materials we perform 2 additional types of electrochemical experiment. In pH 13 electrolyte without a hole scavenger, where the photoanodes 
were found to exhibit little to no photoactivity in Figure 1, cyclic voltammetry (CV) at $0.05 \mathrm{~V} \mathrm{~s}^{-1}$ was perform from just below the OER Nernstian potential to approximately $0.55 \mathrm{~V}$ overpotential. The electrolyte contact area was approximately $0.5 \mathrm{~cm}^{2}$ but was not precisely measured, so the CV is plotted using the current (not the current density) for representative CVs at 4 library compositions in Figure S6. Among the systematic trends with respect to composition, the most notable are the increase in both double layer capacitance and the OER electrocatalytic activity with $\mathrm{Mn}$ concentration. When the $\mathrm{Mn}_{2} \mathrm{O}_{3}$ phase is present, catalysis is observed below $0.5 \mathrm{~V}$ overpotential, but the observed electrocatalysis onset for $\mathrm{NiMnO}_{3}$ and its phase mixture with $\mathrm{Ni}_{6} \mathrm{MnO}_{8}$ is above $0.5 \mathrm{~V}$ overpotential, which is commensurate with the introductory discussion of previous reports of detectable but not high activity of these materials, and with the requirement for adding a hole scavenger to attain appreciable photocurrent since the surface does not contain a suitable electrocatalyst.

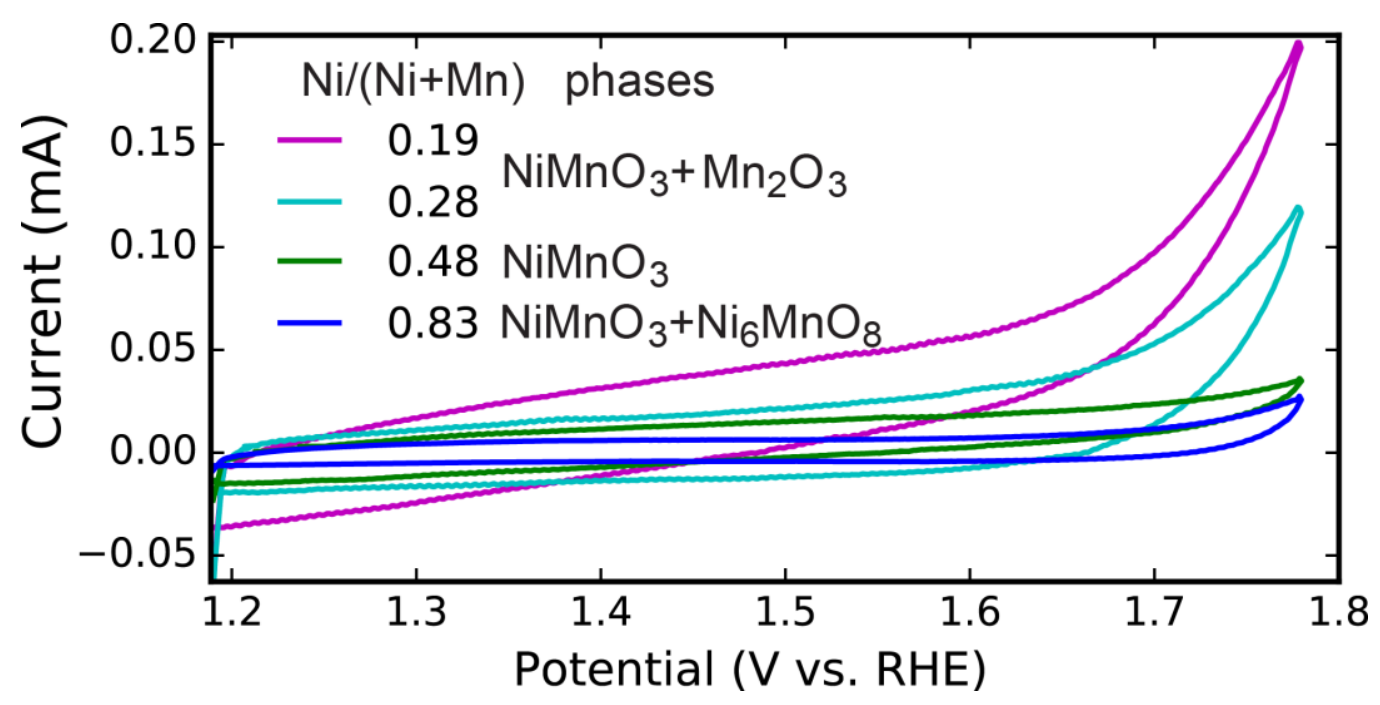

Figure S6. CVs with no illumination for select compositions in $\mathrm{pH} 13$ electrolyte $0.05 \mathrm{~V} \mathrm{~s}^{-1}$ revealing the relative catalytic inactivity of $\mathrm{NiMnO}_{3}$ and its phase mixture with $\mathrm{Ni}_{6} \mathrm{MnO}_{8}$, requiring a hole scavenger to be present to attain photoactivity with $1.23 \mathrm{~V}$ vs RHE bias.

Due to the high absorption coefficient, thinner Ni-Mn oxide thin can still absorb sufficient light and may provide higher photocurrent depending on the limiting factors in the thicker films. To explore this possibility, a film with approximately one third the thickness $(42 \mathrm{~nm})$ was deposited at the $\mathrm{Ni}_{0.64} \mathrm{Mn}_{0.36}$ oxide composition, which as expected exhibited a mixture of $\mathrm{NiMnO}_{3}$ and $\mathrm{Ni}_{6} \mathrm{MnO}_{8}$. The toggled $385 \mathrm{~nm}$ illumination in sulfite-containing $\mathrm{pH} 13$ electrolyte was performed (same conditions as Figure 2), and a linear sweep voltammetry at $0.02 \mathrm{~V} \mathrm{~s}^{-1}$ was used to characterize the photocurrent as a function of applied bias, as shown in Figure S7. The photocurrent at $1.23 \mathrm{~V}$ vs RHE is similar to that observed in the mixed-phase samples in Figure 2, indicating that the thinning of the photoanode did not improve photoactivity, suggesting that a property such as low hole transport or high recombination rate is limiting the photocurrent in these materials. 


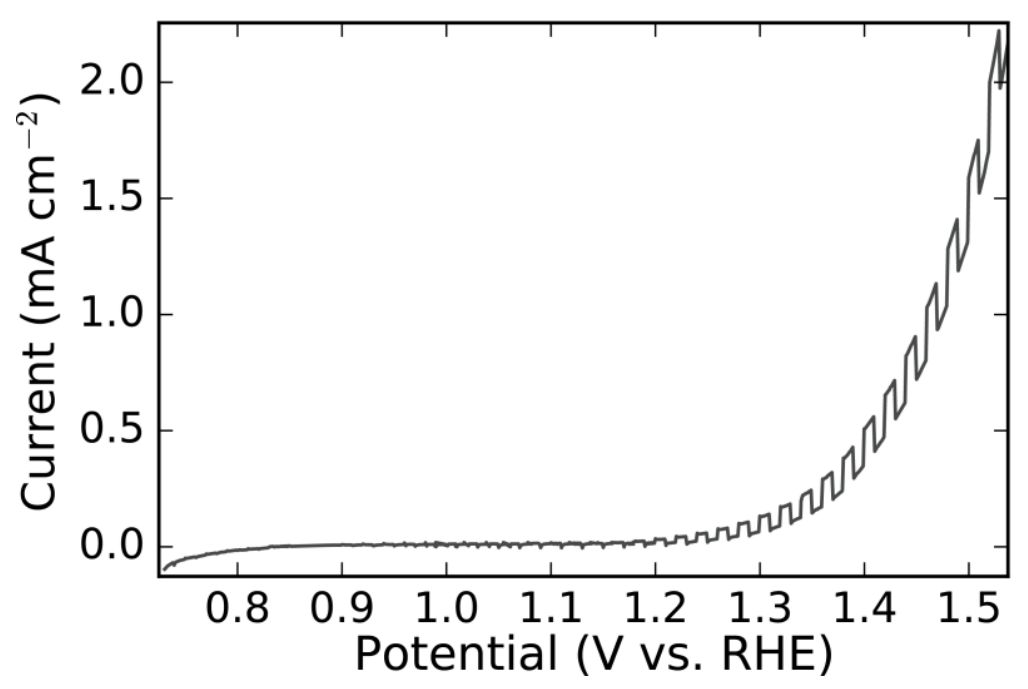

Figure S7. Linear sweep voltammogram at $0.02 \mathrm{~V} \mathrm{~s}^{-1}$ with toggled $385 \mathrm{~nm}$ illumination in $\mathrm{pH} 13$ electrolyte with sodium sulfite hole scavenger on a $\mathrm{Ni}_{0.64} \mathrm{Mn}_{0.36}$ oxide, revealing photoactivity at $1.23 \mathrm{~V}$ vs RHE similar to that observed in Figure 2 . The photocurrent increases dramatically with increased bias voltage, and the sulfite also oxidizes in the dark at potentials above $1.23 \mathrm{~V}$ vs RHE.

\section{Computational details}

DFT computations are performed using the Vienna software package (VASP) ${ }^{2,3}$ with the PAW pseudopotentials ${ }^{4}$, the generalized gradient approximation (GGA) as implemented by Perdew, Burke and Ernzerhoff (PBE) ${ }^{5}$, and the screened hybrid functional of Heyd, Scuseria, and Ernzerhof (HSE) ${ }^{6}$. We study the dependence of band gaps and band edge energies on the mixing parameter for the HartreeFock exchange potential which is in a range from $10 \%$ to $25 \%$. We adopt a 6x6x6 Gamma-centered kpoint mesh for the integrations over the Brillouin zone. Spin-polarization is included in all calculations with ferrimagnetic magnetic configurations for $\mathrm{Mn}$ atoms in $\mathrm{NiMnO}_{3}$ and ferromagnetic orderings for those in $\mathrm{Ni}_{6} \mathrm{MnO}_{8}$. The Hubbard $\mathrm{U}$ value for $\mathrm{Mn}$ and $\mathrm{Ni}$ used in the $\mathrm{PBE}+\mathrm{U}$ surface slab calculations are adopted from the previous work. ${ }^{7}$ An energy cutoff of $450 \mathrm{eV}$ is used for the HSE bulk calculations and $\mathrm{PBE}+\mathrm{U}$ surface slab calculations.

\section{$\mathrm{Ni}_{6} \mathrm{MnO}_{8}$ electronic structure and implications for heterojunction with $\mathrm{NiMnO}_{3}$}

As noted in the communication, an HSE mixing parameter of $14 \%$ provides quantitative agreement between the HSE and measured direct band gaps. To characterize the nature of the heterojunction described in the communication, HSE calculations with the same mixing parameter were performed on $\mathrm{Ni}_{6} \mathrm{MnO}_{8}$. The full band structure was calculated using $\mathrm{PBE}+\mathrm{U}$ and the results are summarized in Figure S8. The $\mathrm{Ni}_{6} \mathrm{MnO}_{8} \mathrm{VBM}$ is at slightly higher energy than $\mathrm{NiMnO}_{3}$ such that a heterojunction would involve hole injection from $\mathrm{NiMnO}_{3}$ to $\mathrm{Ni}_{6} \mathrm{MnO}_{8}$ as illustrated in the figure. Since $\mathrm{NiMnO}_{3}$ is pseudo-direct but $\mathrm{Ni}_{6} \mathrm{MnO}_{8}$ has a much lower $\mathrm{CBM}$ that defines its indirect gap, the nature of the junction appears to be sufficiently complicated that the exact mechanism for the improvement in photocurrent in the phasemixed samples cannot be completely understood from these initial calculations. 

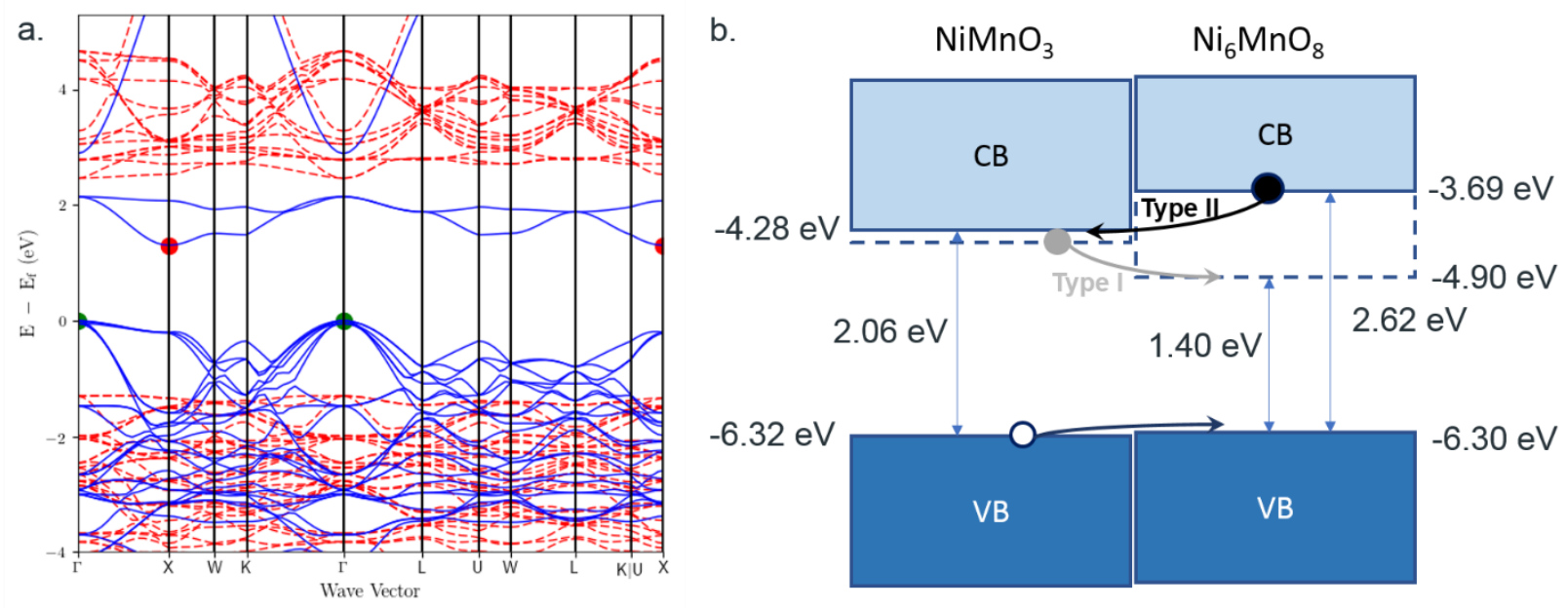

Figure S8. a. Calculated band diagram (PBE+U) for $\mathrm{Ni}_{6} \mathrm{MnO}_{8}$ with spin up and spin down channels denoted with red dashes and solid blue, respectively. The VBM and CBM are noted by green and red points, respectively. b. Band alignment from HSE calculations with $14 \%$ mixing parameter where band gaps are labelled with blue arrows and the other energies refer to band edges with respect to vacuum. The calculated $\mathrm{Ni}_{6} \mathrm{MnO}_{8}$ direct gap of $2.62 \mathrm{eV}$ is similar to the measured direct gap of $2.4 \mathrm{eV}$. The calculated indirect gap of $1.40 \mathrm{eV}$, which has the same VBM an the indirect CBM illustrated with a dashed line, is closer to the lower of the 2 possible indirect band gap values inferred from the Tauc signal in Figure S3.

\section{Structural details for $\mathrm{NiMnO}_{3}$ and $\mathrm{Ni}_{6} \mathrm{MnO}_{8}$}

The $\mathrm{NiMnO}_{3}$ and $\mathrm{Ni}_{6} \mathrm{MnO}_{8}$ crystal structures are illustrated in Figure 3 and are space group 148 and 225, respectively. The lattice constants of relaxed models with the $14 \%$ mixing parameter are $0.5334 \mathrm{~nm}$ and 0.5919 $\mathrm{nm}$, respectively, in good agreement with the ICSD values of 0.5343 and 0.5883 , respectively. The calculated lattice angle of $\mathrm{NiMnO}_{3}$ is $54.66^{\circ}$, which is also in excellent agreement with the experimental value of $54.65^{\circ}$. The angles for $\mathrm{Ni}_{6} \mathrm{MnO}_{8}$ is $60^{\circ}$ by definition. The ICSD values are obtained from Materials Project, $\mathrm{NiMnO}_{3}$ with $\mathrm{mp}-19331$ and $\mathrm{Ni}_{6} \mathrm{MnO}_{8}$ with mp-19442. 


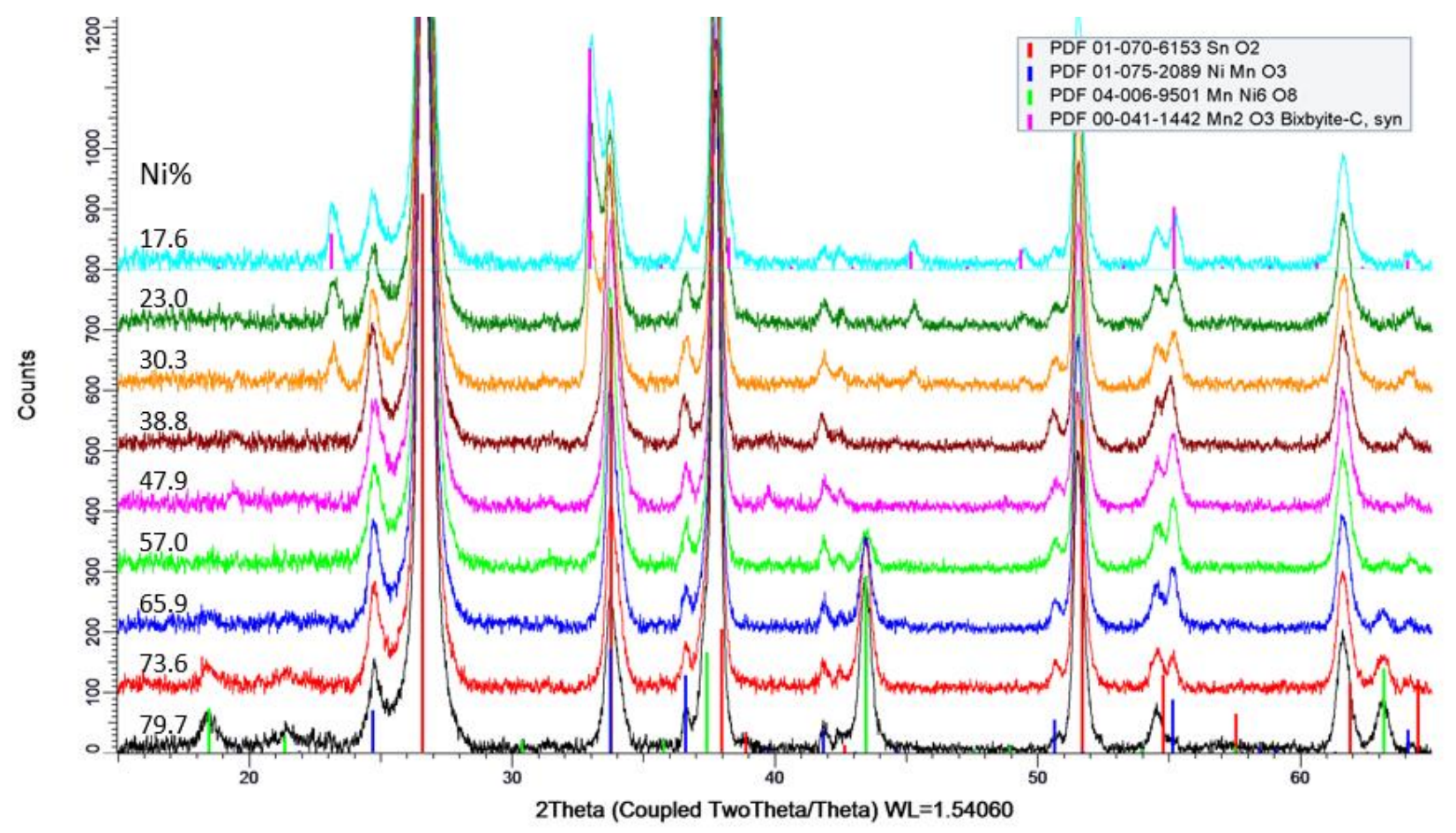

Figure S9. Integrated 1D XRD patterns along the thin film compositions, which were used to derive composition-dependent phase behavior shown in Figure $1 \mathrm{c}$ in the main text. Three phases were identified: $\mathrm{Mn}_{2} \mathrm{O}_{3}$ (ICDD PDF: 00-041-1442), NiMnO 3 (ICDD PDF: 01-075-2089) and $\mathrm{Ni}_{6} \mathrm{MnO}_{8}$ (04-006-9501). No differences were observed between the measured XRD pattern and ICDD stick patterns. With increasing $\mathrm{Ni}$ content, the phases were the mixture of $\mathrm{Mn}_{2} \mathrm{O}_{3}$ with $\mathrm{NiMnO}_{3}$ to phase pure $\mathrm{NiMnO}_{3}$, and further became the mixture of $\mathrm{Ni}_{6} \mathrm{MnO}_{8}$ and $\mathrm{NiMnO}_{3}$. Since there is no evidence of any structural change of $\mathrm{NiMnO}_{3}$ phase, the observed improvement of PEC performance is due to the mixed phases of $\mathrm{Ni}_{6} \mathrm{MnO}_{8}$ and $\mathrm{NiMnO}_{3}$.

The grain size of $\mathrm{Ni}_{6} \mathrm{MnO}_{8}$ and $\mathrm{NiMnO}_{3}$ phases is approximately $17 \mathrm{~nm}$ and $21 \mathrm{~nm}$, respectively. They were calculated using (400) diffraction peak at 2 theta $=43.47^{\circ}$ for $\mathrm{Ni}_{6} \mathrm{MnO}_{8}$ and (110) peak at 2 theta $=36.61^{\circ}$ for $\mathrm{NiMnO}_{3}$. Within the resolution of the measurement, no deviations were observed from either database peak pattern, indicating that the lattice constants and bond angles noted above are representative of these phases as they exist in the thin film photoanode samples.

\section{Mott-Schottky analysis}

Mott-Schottky analysis (MS-A) was carried out in the same $\mathrm{pH} 13$ electrolyte as used in the other experiments. MSA was performed using an o-ring sealed cell enclosing approximately $0.25 \mathrm{~cm}^{2}$ of the thin film library in the phasepure $\mathrm{NiMnO}_{3}$ region. MS-A was done at a constant frequency of $5.5 \mathrm{kHz}$ and $20 \mathrm{mV}$ RMS between 1.1 to $1.8 \mathrm{~V}$ vs. RHE in $50 \mathrm{mV}$ steps. Due to the imperfect nature of the coating two capacitors C1 and C2 were assumed to be in parallel to model a Faradaic and space charge contribution. From this MS-A the flatband is estimated to be between 1.1 and $1.2 \mathrm{~V}$ vs. RHE. The onset of photocurrent in Figure S7 is approximately $1.1 \mathrm{~V}$ vs RHE, in reasonable agreement with the MS-A result. Assuming the relative dielectric constant of $\mathrm{NiMnO}_{3}$ between $5.7\left(\mathrm{NiO}^{8}\right)$ and 64 $\left(\mathrm{MnO}_{2}{ }^{9}\right)$ the charge carrier density is on the order of $10^{13}$ to $10^{14} \mathrm{~cm}^{-3}$, indicating that the semiconductor is not degeneratively doped. This flatband potential is far higher than desirable for solar photochemistry applications, and comparison with the band energy calculations of Figure 4 indicates that the Fermi level is closer to the VBM than CBM, counter to the standard picture of the band energetics of an n-type 
semiconductor. Further study will be required to fully characterize band energetic in this material, and in particular whether there is a mid-gap state that is pinning the Fermi level and whose elimination would result in a major advance in the development of nickel manganates for solar photochemistry.

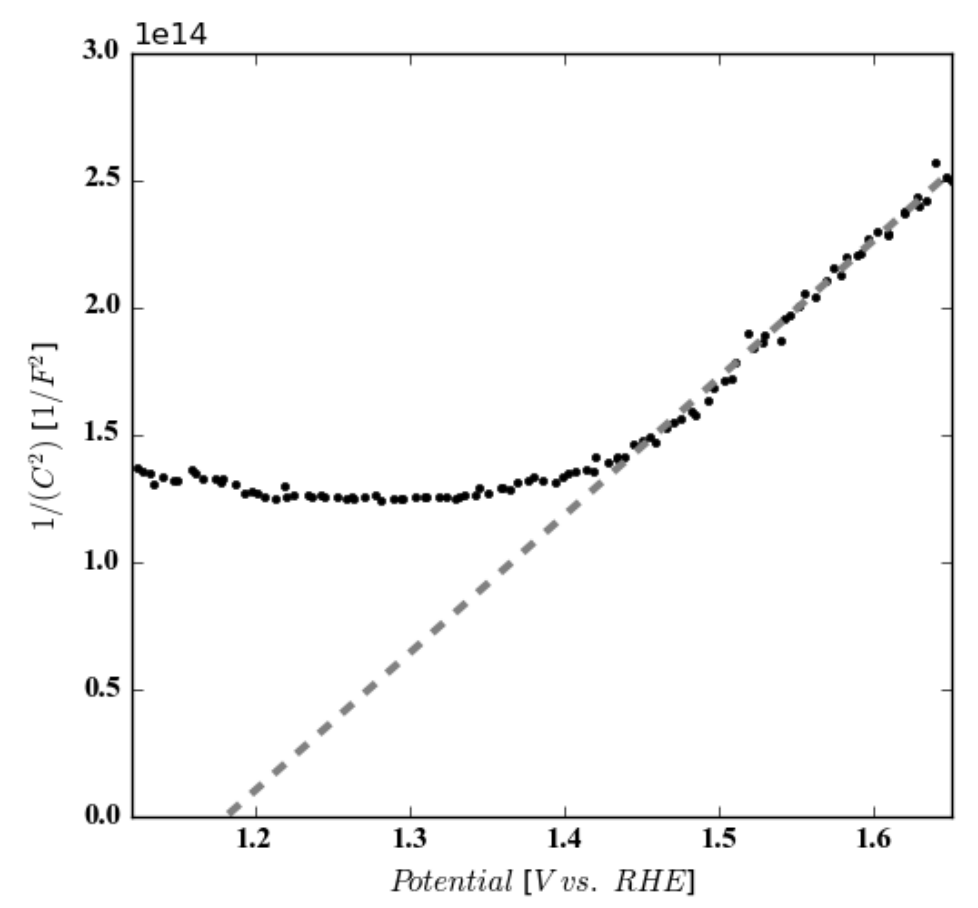

Figure S10. Mott-Schottky analysis assuming two parallel capacitor circuits. The material is n-type with a flatband potential of 1.1-1.2 V vs. RHE and a charge carrier density extracted from the slope Mott-Schottky slope indicated by the dashed grey line of $10^{13}$ to $10^{14} \mathrm{~cm}^{-3}$.

\section{References}

1. A. Shinde, S. K. Suram, Q. Yan, L. Zhou, A. K. Singh, J. Yu, K. A. Persson, J. B. Neaton and J. M. Gregoire, ACS Energy Letters, 2017, DOI: 10.1021/acsenergylett.7b00607, 2307-2312.

2. G. Kresse and J. Furthmuller, Comp Mater Sci, 1996, 6, 15-50.

3. Q. Yan, G. Li, P. F. Newhouse, J. Yu, K. A. Persson, J. M. Gregoire and J. B. Neaton, Adv. Energy Mater., 2015, 5, 1401840.

4. P. E. Blochl, Phys Rev B, 1994, 50, 17953-17979.

5. J. P. Perdew, K. Burke and M. Ernzerhof, Phys Rev Lett, 1996, 77, 3865-3868.

6. (a) J. Heyd, G. E. Scuseria and M. Ernzerhof, J Chem Phys, 2003, 118, 8207-8215; (b) J. Heyd, G. E. Scuseria and M. Ernzerhof, Journal of Chemical Physics, 2006, 124, 219906.

7. A. Jain, G. Hautier, S. P. Ong, C. J. Moore, C. C. Fischer, K. A. Persson and G. Ceder, Phys Rev B, 2011, 84.

8. L.-H. Ye, N. Luo, L.-M. Peng, M. Weinert and A. J. Freeman, Physical Review B, 2013, 87, 075115.

9. F. K. Tan, J. Hassan, Z. A. Wahab and R. a. S. Azis, Results in Physics, 2016, 6, 420-427. 